\title{
AITERIDAD El significado de la práctica docente, en voz de sus protagonistas
}

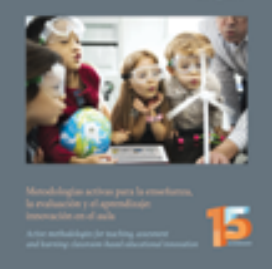

\section{The meaning of teaching practice, in the voice of its protagonists}

Dra. Celia Gabriela Villalpando es docente-investigador de la Universidad Autónoma de Ciudad Juárez (México) (celia.villalpando@uacj.mx) (http://orcid.org/0000-0001-9571-6308)

(1) Dra. María Armida Estrada-Gutiérrez es docente-investigador de la Universidad Autónoma de Ciudad Juárez (México) (marmida@uacj.mx) (http://orcid.org/0000-0003-3689-343X)

(D) Dr. Guillermo Alberto Álvarez-Quiroz es docente-investigador de la Universidad Autónoma de Ciudad Juárez (México) (guillermo.alvarez@uacj.mx) (http://orcid.org/0000-0002-9621-4155)

Recibido: 2020-01-31 / Revisado: 2020-05-27 / Aceptado: 2020-06-04 / Publicado: 2020-07-01

\section{Resumen}

La existencia del presente artículo obedece a la necesidad apremiante por parte de los profesionales de la educación de poner en la mesa de discusión sus experiencias y saberes, a fin de reconstruir su pensamiento pedagógico. El presente documento, en un intento por conocer desde la empírea lo dicho hasta el momento sobre su ocupación, busca recuperar su voz y con ello aportar conocimiento al área de la práctica docente, razón por la cual se plantean como objetivos de investigación: Conocer los aspectos que conforman el ser y el quehacer en esta actividad; interpretar los significados que construyen acerca de su actuación pedagógica; así como comprender la construcción de su saber pedagógico en relación con su labor. La metodología de investigación se aborda desde una postura cualitativa, mediante el estudio de caso, este posibilita la revisión exhaustiva de 43 maestros estudiantes de diferentes generaciones que cursaron la materia de «Análisis de la Práctica Docente» de un programa de Maestría en Ciudad Juárez, Chihuahua. Uno de los resultados encontrados, rescata que estos actores en cuestión saben que su ejercicio involucra sus percepciones sobre el entorno que le rodea, entorno impregnado de múltiples interacciones. Como conclusión, el análisis crítico y reflexivo de su actuar requiere tanto de un diálogo colectivo como de una interpretación de acciones.

Descriptores: Práctica docente, docencia, estudio de caso, saberes docentes, profesionales de la educación, investigación cualitativa.

Forma sugerida de citar: Villalpando, C., Estrada-Gutiérrez, M., \& Álvarez-Quiroz, G. (2020). El significado de la práctica docente, en voz de sus protagonistas. Alteridad, 15(2), 229-240. https://doi.org/10.17163/alt.v15n2.2020.07

\section{Abstract}

This article stems from the education professionals' pressing need to bring up their experiences and knowledge at the discussion table in order to reshape their pedagogical thinking. In an attempt for the empirical sphere to inform what has been said thus far about the teaching practice, this document seeks to collect the educators' voices, thereby contributing new knowledge to the teaching field. Thus, the proposed objectives of this research are: To know the aspects that make up the essence and the tasks involved in the teaching occupation; to interpret the meanings that educators give to their pedagogical performance; and to understand their construction of their own pedagogical knowledge in relation to their work. The study followed the type of qualitative methodology known as case study, which allowed for a detailed, in-depth examination of 43 student teachers from different generations who took the subject of «Teaching Practice Analysis» as part of a master's degree program in Ciudad Juarez, Chihuahua, Mexico. One of the results found showed that the subjects involved know that their praxis involves their way of thinking, reasoning and valuing the world and that it is also highly influenced by multiple interactions. The research concluded that critical and reflective analysis of teachers' actions requires both a collective dialogue and an interpretation of their actions.

Keywords: Teaching practice, teacging, case study, teaching knowledge, education professionals, qualitative research. 


\section{Introducción y estado de la cuestión}

"Desde que México ingresó a la OCDE en 1994, las reformas educativas han sido constantes... Sin embargo, la crisis educativa se fue profundizando, impactando de manera negativa a la población mexicana en edad de estudiar" (López, 2013, p. 61). Como consecuencia, en febrero de 2013, la reforma educativa mexicana fue declarada constitucional por el poder legislativo federal y en el mismo mes fue promulgada y publicada por el ejecutivo federal. Cabe señalar que tal crisis educativa no es responsabilidad exclusiva de la docencia, no obstante, corresponde al profesional de la educación revisar y realizar una lectura de su quehacer, a fin de visibilizar su actuar con respecto a la mencionada crisis. Al respecto, Rodríguez (2016) afirma que todo este entramado llamado educación no puede ser abordado desde la pasividad y el desconocimiento del docente, pues requiere mayor responsabilidad de tu parte.

Por ello, la revisión del quehacer docente demanda el estudio de los aspectos que conforman la práctica, atomizándolos para así lograrlo, pero sin olvidar que son parte de una totalidad. Este análisis conlleva explorar sus áreas de oportunidad y sus fortalezas para ubicarse y reconocerse dentro de este acto educativo (Fierro et al., 1999, p. 26), lo cual permite una nueva lectura de esta realidad, con una visión más amplia del proceso educativo; lectura que pretende generar una transformación en la práctica y provocar un crecimiento personal y profesional, lo cual se puede traducir en resignificarla, y así recuperar tanto el valor como el significado que tiene la profesión. En este sentido, se hace necesaria la presencia de la práctica reflexiva, que según Domingo (2013), se traduce como aquella actitud metódica e intencionada indispensable para el profesional de la educación. Tales espacios y sus dinámicas generan el interés por abordar una investigación que logre comprender este ejercicio educativo desde la óptica del profesorado, a través de la revisión, análisis y reflexión de su práctica cotidiana, así como de la construcción de su saber pedagógico. Por lo que se parte de la idea que dicho quehacer está impregnado de interacciones y situaciones peculiares, diferentes y únicas, lo que lo hace complejo de entender. Para destacar, los participantes en este proceso indagatorio son 43 profesores estudiantes de diferentes generaciones que cursaron la materia de «Análisis de la Práctica Docente» de un programa de Maestría en Ciudad Juárez, Chihuahua, quienes según Terrón (2019), leen, escriben y reflexionan sobre lo que piensan y hacen de su profesión, a fin de "objetivar las decisiones sobre el oficio" (p. 169).

De acuerdo con El Sahili (2011) la profesión educativa es complicada debido a todos los aspectos que giran alrededor de ella, y sobre todo a los que se relacionan con las problemáticas docentes; aunado a ello, están "las competencias personales [concebidas] como eje transversal del perfil competencial en tanto se asocian a la capacidad del docente como sujeto, de movilizar e integrar sus recursos cognitivos y motivacionales en la regulación de la calidad de su desempeño profesional" (González et al., 2017, p. 133); de manera que efectuar este análisis conlleva a la confrontación de conocimientos y convicciones.

Para realizar una revisión consciente, en un primer momento se busca reconocer al docente como individuo, con una particular historia de vida donde se posibilita la oportunidad de abordar una reflexión del presente y futuro del profesor. En otro plano, se identifica que la labor del maestro es una acción colectiva, pues de manera recíproca, el profesional de la educación aporta a la institución - como ese espacio idóneo para la socialización- sus saberes, ideas, conocimientos y experiencia.

Evidentemente, este ejercicio está impregnado de interacciones interpersonales, pues se hacen presentes relaciones con alumnos, otros maestros, directivos y padres de familia, mismas que por su propia naturaleza tienden a ser complejas. El clima institucional se genera en el entramado donde se tejen dichas relaciones, lo cual impacta en la actua- 
ción de la docencia, pues se ponen en juego tanto la actuación como las decisiones para las diversas actividades. A nivel social, "la práctica docente intenta recuperar un conjunto de relaciones que se refieren a la forma en que cada docente percibe y expresa su tarea como agente educativo cuyos destinatarios son diversos sectores sociales" (Fierro et al., 1999, p. 33), esto recupera cualquier aspecto o ámbito que exigen un determinado tipo de profesor. En otras palabras, es reflexionar sobre el sentido de su labor con respecto al momento histórico que está viviendo, realidad que lo ubica en realidades opuestas en relación con otros profesionales de la educación.

Inevitablemente, a fin de abordar el quehacer del docente, es esencial considerar lo referente a la didáctica, esto es, pensar sobre la manera en la que cada maestro decodifica y codifica los saberes para ser trabajados con y por sus estudiantes, sobre la forma como conduce las situaciones didácticas en este espacio educativo, tal como los problemas académicos que enfrentan. Además, es menester de la revisión abordar el aspecto valoral, pues se reconoce que la labor de este profesional contiene una referencia axiológica; ya que pone en juego, todo su ser (valores, actitudes y conocimientos) dentro de la dinámica educativa, reconociendo con ello la influencia que tiene el docente sobre la manera en la que pudiera interpretar las realidades que viven sus estudiantes (Fierro et al., 1999).

Aunado a ello, el profesor tiene que ver con los valores institucionales que definen las directrices del sistema educativo, que a su vez están presentes en los planes de estudio, los cuales sirven para diseñar sus situaciones didácticas, de igual manera permite examinar la vida cotidiana de la institución, con la intención de reconocer el tipo de valores que se están viviendo en el plantel. Con base en lo expresado, se hace necesario abordar teóricamente los siguientes aspectos:

\subsection{La práctica docente y su conceptualización}

En congruencia con los argumentos recién presentados, interesa partir, a fin de situar el con- cepto en la dimensión correcta, de la aportación realizada por Davini (2015), quien afirma que:

Cuando hablamos de «prácticas» no nos estamos refiriendo exclusivamente al desarrollo de habilidades operativas, técnicas o para el «hacer», sino a la capacidad de intervención y de enseñanza en contextos reales complejos ante situaciones que incluyen distintas dimensiones y una necesaria reflexión, a la toma de decisiones $y$, muchas veces, hasta al tratamiento contextualizado de desafíos o dilemas éticos en ambientes sociales e institucionales. En otros términos, en las prácticas se trata con situaciones y problemas genuinos. (p. 29)

Al respecto, se intenta persuadir al lector de evitar - en este documento- reducir dicha actividad a ese simple «hacer»; contrario a ello, incita a reconocer las dimensiones o elementos que la conforman para llegar a comprenderla en su totalidad; al respecto, Fierro et al. (1999) afirman que "la práctica docente [es considerada] como una praxis social, objetiva e intencional en la que intervienen los significados, las percepciones y las acciones de los agentes implicados en el proceso" (p. 21), en este mismo tenor, Vergara (2016) complementa que además de ser dinámica, contextualizada y compleja, dicha praxis posee un aspecto característico "un agente [que] ejerce su actividad sobre determinada realidad" (p. 75). Aunado a esto, las condiciones actuales en las que se desarrolla la educación, sumada a los elementos subjetivos que dan vida al actuar de cada una de las personas partícipes del proceso educativo, la hacen ser un ejercicio social multideterminado. Este es entonces, "una práctica intencional... que implica las acciones realizadas por el profesor para «facilitar» que el educando se eduque" (Loredo et al., 2008, p. 4).

A modo de colofón, esta labor trasciende la idea de pensar simple y llanamente en el actuar del profesional de la educación, pues, además de ello, se impregna de matices que ofrecen los contextos institucionales, políticos, sociales, organizativos y económicos; al igual que de las características de 
los propios estudiantes, compañeros maestros, directivos y su particular forma de ser, todo ello, se interpreta como un entramado de vidas que confluyen, construyen y reconstruyen la actividad que le compete al profesional de la educación.

\subsection{Significado de la actuación docente}

La actuación del sujeto en cuestión trasciende las cuatro paredes del aula, pues su ejercicio también se hace presente en todos y cada uno de los espacios institucionales donde labora; además, está impregnado de experiencias, creencias y saberes tanto teóricos como empíricos, mismos que le permiten comprender el mundo donde se desenvuelve; en otras palabras, pone en juego una serie de construcciones personales y sociales, del mismo modo que un marco de referencia teórico que fundamenta su toma de decisiones. $\mathrm{Al}$ respecto, Sacristán (en Vergara, 2005) expone que:

Cuando se habla de significados, no se hace referencia a la teoría, sino a los saberes, creencias, valores que ayudan a prefigurar el hacer que un profesor realiza como docente, ya que se puede decir que, de acuerdo a como el educador «signifique» su práctica, es la forma como la realiza. (p. 692)

Razón por la cual, como bien se mencionó, más allá de interesarse exclusivamente por el quehacer, atrae la atención conocer la estructura de creencias, supuestos e intenciones que fundamentan su actuar; aun cuando "analizar la realidad producida por la intervención de procesos subjetivos e identificar cómo los docentes... [realizan su] práctica y cómo aportan sus propios significados, no es tarea fácil" (VillalpandoSifuentes, 2015, p. 92), sin embargo, con ello se alcanza una comprensión de su labor y el significado de la misma. En este sentido, se asume el entorno donde labora como un referente para su proceder, pues el escenario institucional, las circunstancias que lo envuelven, así como los cambios y la incertidumbre propia de la profesión, son algunos de los muchos ingredientes que van construyendo la esencia de dichos significados.

Por lo que se puede decir, el significado de la práctica docente se encuentra íntimamente relacionado con los aspectos y realidades que lo enmarcan, pues en palabras de Evangelista (2015), los profesores parten de su quehacer para enlazarlo con diferentes aspectos de la realidad social, a fin de construir la representación de su ejercicio. Este sujeto en su saber, en su saber hacer y en su querer hacer, se encuentra inmerso en un sinfín de presupuestos que va generando a lo largo de su profesión y ello obedece a la naturaleza subjetiva de su labor y al carácter social de los contextos mencionados.

Entonces, en un intento por llegar a la comprensión de las acepciones edificadas por el profesional de la educación, no solo se requiere de la revisión honesta de las creencias, saberes o intuiciones que subyacen en su biografía profesional, tampoco de la manera de abordar la acción pedagógica (Montanares \& Junod, 2018); sino de ser capaz de enmarcar y conectar las realidades de su historia personal, del contexto histórico donde se desempeña y de los hechos educativos y esquemas pedagógicos encaminados a dar vida a su quehacer; aristas de la educación que reconocen la incertidumbre y la confusión generada por el profesor a lo largo de su carrera magisterial.

\subsection{Construcción de saberes pedagógicos}

Sin lugar a duda, las instituciones educativas son uno de los principales espacios donde el profesorado construye sus saberes pedagógicos, no obstante, se hacen presentes, escenarios que asisten en dicha construcción; la cual a lo largo de la vida profesional se va reconstruyendo, esto a través de las diversas interacciones que experimenta. De acuerdo con Díaz (2006), "el saber pedagógico son los conocimientos, construidos de manera formal e informal por los docentes; valores, ideologías, actitudes, prácticas" (p. 95); todo aquello que surge de sus interacciones. La 
dirección que lleva la edificación de estos saberes se encamina hacia el conocimiento científico requerido para dar vida a los contenidos escolares, de igual manera, al desarrollo de la experticia necesaria en el uso de estrategias didácticas, en el manejo del currículum con el que trabaja; así como del conocimiento indispensable de sus estudiantes y sus procesos de aprendizaje. Según Díaz (2006), los profesores, independientemente de que lo hagan de manera intencionada o no, diariamente crean teoría, la cual podría aportar al entendimiento de su ejercicio profesional.

Por otro lado, se puede "llamar conocimiento del cuerpo docente a aquel que surge del intercambio y la reflexión profesional a partir de procesos de observación y discusión de la práctica y que tienen como resultado la valoración del impacto buscado de las prácticas educativas" (Minakata, en Verástegui, 2019, p. 171); en este sentido, sería errado dejar de lado la idea de concebir al saber pedagógico como una construcción social de la realidad, que atiende diferentes intereses tanto a nivel personal, político, educativo como social; pues el profesorado además de ejercer su ejercicio frente a grupo, a lo largo de su trayectoria profesional se encuentra inmerso en diferentes contexto - llámese congresos, talleres, reuniones, etc. - rodeado de personas que, de igual manera, coinciden con éste para abordar temáticas relacionadas con la educación. Estas interacciones se reconocen como los espacios potencialmente poderosos para la construcción de los saberes pedagógicos, pues representan una invaluable herramienta tendiente a configurar y reconfigurar estos últimos (Hurtado et al., 2015).

En este mismo sentido, Torres, et al. (2014), afirman que además de que estas responsabilidades propias de la profesión van en aumento, se están redefiniendo atendiendo nuevos desafíos, mismos que obedecen a los constantes cambios que se viven en la actualidad; por lo que los conocimientos generados posibilitan una nueva lectura de la realidad educativa, lo cual reviste mayor importancia en la práctica docente, ya sea dentro de las tomas de decisiones en el plano pedagógico o en la actuación a nivel institucional; ello implica el desarrollo de un proceso reflexivo de los saberes cimentados en todos y cada uno de estos espacios; los cuales constituyen la base que permite comprender su actuar.

\section{Metodología}

Se pretende comprender el quehacer docente como un proceso dinámico y multifactorial, y es a partir de tal enunciación que se opta por elegir, para guiar este proceso de investigación, la metodología cualitativa "como escenario de actividades interpretativas" (Denzin \& Lincoln, 2011, p. 55), esta, en congruencia con el paradigma interpretativo que se aborda desde la axiología, epistemología, ontología y metodología (Denzin \& Lincoln, 2012), ya que su finalidad radica en el conocimiento del individuo en toda su complejidad subjetiva, además se busca "reconocer que el conocimiento de la realidad humana supone no solo la descripción operativa de ella, sino ante todo la comprensión del sentido de la misma por parte de quienes la producen y la viven" (Sandoval, 2002, p. 39); en otras palabras, se procura la comprensión desde la visión del otro (Quecedo \& Castaño, 2002); objetivo que persigue esta investigación, misma que se guía a través de un estudio de caso, el cual "implica un proceso de indagación que se caracteriza por el examen detallado, comprehensivo, sistemático y en profundidad del caso objeto de interés" (Rodríguez et al., 1996, p. 92). Para este documento, los casos a estudiar son el total de la población - 43 maestrantes - de los grupos que han tomado el curso de "Análisis de la Práctica Docente», en un lapso de un año y medio, del primer semestre de un programa de posgrado en Ciudad Juárez, México.

Obedeciendo al trabajo de reflexión y análisis que implica la revisión de la actividad del profesional de la educación, mediante dicha metodología, el diálogo juega un rol preponderante, al respecto, Fierro et al. (1999), afirman que el diálogo realizado constantemente 
va generando la crítica dentro del pensamiento; razón por la cual en el transcurso de las clases en mención, dicha actividad es protagonista en el desarrollo de las técnicas empleadas con la intención de recuperar información, por ejemplo de las entrevistas y los grupos focales. Dichas técnicas se eligieron porque tienen como fin que los docentes pongan en la mesa de discusión sus experiencias y saberes, reconstruyendo con ello su pensamiento pedagógico, teniendo siempre en mente que "el docente es un sujeto en construcción” (Tovar \& García, 2012, p. 885).

En el caso de la entrevista, Rodríguez et al. (1996) mencionan que es considerada como esa relación entre dos sujetos quienes establecen una comunicación con base en un tema en particular, en este caso, sobre el análisis de la propia práctica, tal interacción se desarrolla en diferentes espacios escolares entre los investigadores y los maestrantes partícipes. Por otro lado, se realiza una acción similar, pero en pequeños grupos, la cual Leiner (2005), reconoce como grupo focal; este es una especie de entrevista, pero entre el investigador (quien funge como moderador) y una docena de sujetos estudiados; quienes al abordar la misma temática facilitan información fundamental para el desarrollo de esta investigación.

Cabe señalar que desde el ámbito cualitativo, al investigador le atrae la multiplicidad de realidades de los sujetos estudiados; a fin de abordarlas y conocerlas echa mano de la triangulación (Denzin \& Lincoln, 2013); por ello, con el propósito de comprender desde la óptica del profesorado dicha práctica, y en atención a la necesidad de garantizar la validez de este estudio, se hace uso de la triangulación de datos, la cual, como técnica posibilita un ejercicio de cotejo y balance entre la diversidad de información recogida (Rodríguez et al., en Aguilar \& Barroso, 2015), pues es la idónea para interpretar y comprender este recorte de la realidad.

\section{Resultados}

Con el propósito de abordar el presente apartado, es interesante partir de la idea que Perrenoud (2001) expone:

Una práctica reflexiva no es solamente una competencia al servicio de los intereses legítimos del enseñante, sino que también es una expresión de la conciencia profesional. Los profesores que no reflexionan más que por necesidad y que dejan de plantearse cuestiones desde el momento en que se sienten seguros no son practicantes reflexivos. (p. 48)

Con base en lo expresado, se considera que el producto de esta indagación es inacabado, pues la complejidad inherente al ejercicio que desempeña el profesional de la educación se encuentra en constante movimiento y la enmarca en una realidad de carácter subjetivo, por lo que adquiere relevancia en el terreno de la educación. La discusión puede enriquecerse gracias a que se favorece el proceso comunicativo desde diferentes direcciones, a fin de establecer una reciprocidad de información entre los actores del hecho educativo (Castillo \& Montoya, 2015). A fin de develar lo que el docente construye en torno a su actividad, se hacen tres planteamientos específicos que permiten nutrir la indagación en cuestión; pues de manera cualitativa se manifiesta la relación existente entre dichos significados y el quehacer cotidiano del profesorado.

\subsection{Ser y quehacer en la práctica docente}

Para los profesionales de la educación estudiados, su quehacer representa ese conjunto de actividades y acciones que desarrollan no solo en el espacio áulico, pues más bien se viene gestando desde el diseño de la planificación hasta el proceso valorativo de su actuación; en otras palabras, su labor se encuentra lejos de limitarse a su «hacer», pues involucra su pensamiento, su razonamiento y su valoración sobre el mundo, 
así como sus nociones y percepciones de lo que es la enseñanza y el aprendizaje, mismas que determinan la implementación de su trabajo en los entornos educativos.

La práctica docente inevitablemente es un elemento fundamental en el hecho educativo; pues con base en las decisiones tomadas, la educación encuentra su rumbo; no obstante, no determina el éxito o fracaso de las mismas; pues habrá que considerar que existen aspectos del entorno político, social, institucional que la afectan; de igual manera se reconocen elementos que brindan los sujetos con quienes trabaja como las familias de sus estudiantes, su contexto, sus historias de vida, además de ello, se encuentran los aspectos imprescindibles en toda dinámica escolar como la carga curricular, trámites administrativos y académicos; por lo cual se puede admitir: El quehacer se interpreta como una compleja trama multifactorial que da vida a diversos espacios educativos. Al respecto, Vergara (2005), afirma que, con el propósito de comprender la labor del docente, se requiere conectar los sucesos ocurridos en los entornos educativos desde un panorama global con los ambientes donde se da vida a estos, a fin de darles sentido y dejarlos de ver como solo ese hacer de los profesores. En ese mismo tenor, en la empírea uno de los profesores expresa:

Nuestra labor es muy noble, y a mí me gusta mucho, pero hay veces que me siento abrumado por la cantidad de cosas que tenemos que hacer, mucho a nivel administrativo y otro tanto que no tiene nada que ver con lo académico o lo educativo; o sea, además de la carga pedagógica que nos corresponde dentro del aula, debemos considerar otros aspectos que anclan con nuestra labor, que tiene que ver con las nuevas políticas educativas, y pues ni modo, a darle. (D2)

En suma a lo expresado, los maestrantes en esta investigación coinciden en que en el ser y el quehacer dentro de su actuación se encuentran aspectos indispensables en el proceso educativo, como lo son el compromiso, responsabilidad, dedicación, actualización, al igual que el amor y la competencia emocional; además reconocen que su labor tiene diferentes matices, propios de su personalidad y del nivel educativo donde labora; empero, la práctica en cuestión va más allá de ello, pues toca aspectos de las políticas públicas y reformas educativas que han llegado a ser incongruentes con las realidades escolares.

\subsection{Significados de la actuación pedagógica en el espacio áulico}

A partir de las experiencias, vivencias, la historia y las percepciones personales de cada maestro, aunado a las decisiones y actuaciones profesionales, es que la práctica tiene sentido y puede otorgar significado, no obstante, se reconstruye cuando se comparte, se regula y se reflexiona con otros maestros, tal como se manifiesta en este comentario:

Pues indiscutiblemente, pienso que nuestra actuación pedagógica tiene que ver en gran medida con las decisiones que tomamos no solo de manera individual dentro de las aulas, sino con los compañeros, con el director, y esto va desde las reuniones que tenemos programadas, hasta las pláticas informales que tenemos en los pasillos, lo que me hace pensar que eso dice mucho del compromiso que tenemos para con nuestros alumnos y alumnas. Es que siempre estamos pensando cómo hacerle con fulanito que falta mucho, o con zutanito que no entiende esto. (D39)

Para compaginar lo expresado, en palabras de Vergara (2005), el docente "basa sus acciones en el significado de las cosas de su mundo, se considera que es ahí donde «construye» el significado a partir de las interacciones sociales que tiene" (p. 685), sin obviar sus procesos de reflexión e interpretación. Cada decisión que el educador toma para desarrollar su labor obedece al significado que da a su quehacer, a la educación, a la enseñanza, al aprendizaje, a los 
procesos evaluativos, asimismo, a las relaciones que se desarrollan en los espacios áulicos e institucionales, por lo tanto, se puede decir que dichos constructos determinan su actuación con respecto a su compromiso profesional, por lo que sus acciones tienen una razón de ser.

Como ya se ha venido manejando, el quehacer docente está regido por las ideas y creencias de los maestros con respecto a lo que se enseña y a los que se aprende, a lo que Zabalza (2012) opina "interesa considerar no solamente lo que las personas hacen, sino el por qué lo hacen, sobre qué estructuras de creencias, conocimientos o experiencias previas se soporta su plan de acción" (p. 32); en este aspecto se rescata, que los profesores en este estudio, carecen de un edificio teórico sólido para tomar sus decisiones, pues afirman que en la mayoría de las ocasiones solicitan ayuda de sus compañeros para atender problemáticas académicas, y ponen en práctica acciones que en el pasado les fueron útiles, dejando de lado las aportaciones que teóricos o profesionales han ofrecido. Según Vergara (2005) "las acciones que llega a realizar como docente, están determinadas por sus significados, pero además tiene plena consciencia de ello... este es un acto individual que realiza el sujeto para apropiarse de vivencias de manera personal, sin intermediarios" (p. 695).

En definitiva, las decisiones que estos profesionales de la educación han tomado tanto para el desarrollo de su clase como en la inmediatez, están respaldadas por sus experiencias, saberes y hasta por su intuición; cabe señalar que estas decisiones, desde el punto de vista del profesorado, son consideradas las adecuadas para atender cada situación en particular.

\subsection{La construcción del saber ped- agógico en relación con la prác- tica docente}

Dentro de la construcción de los saberes pedagógicos de los maestrantes de este programa de posgrado se encuentra presente, en primera instancia, la teoría adquirida por el maestro para fundamentar la actividad que da vida a la dinámica áulica, teoría que se entrelaza con las concepciones de este con respecto a los aprendizajes de sus alumnos y a su manera de enseñar, esto le permite diseñar, organizar y evaluar las acciones que dirigen dichos procesos. En opinión de Vitarelli (2005):

El campo del saber es un espacio conformado por regímenes discursivos... el caso del saber pedagógico, el concepto de saber nos permite explorar desde situaciones prácticas de la enseñanza, la escuela y el maestro, hasta situaciones conceptuales de la pedagogía. (pp. 11-12)

$\mathrm{Al}$ respecto, los profesores de esta investigación hacen una distinción entre los docentes de formación y los que tienen diferentes perfiles o profesión, opinan que:

Las escuelas formadoras de docentes son las que dan las bases, las herramientas, los cimientos para irnos formando como maestros, con respecto a los compañeros que no tienen formación docente, pues es notorio que les falta algo de didáctica. (D20)

Realidad reflejada en la dinámica áulica; no obstante, al momento de dar vida a los contenidos escolares, se reconoce que el saber pedagógico tiene que ver con la manera como el profesor traduce el «saber científico» en «saber enseñado», a fin de posibilitar que los alumnos logren su comprensión; cabe señalar que, para ello, también se requiere que logre identificar las fortalezas y áreas de oportunidad en la enseñanza y en el aprendizaje. Asimismo, reconocen que los saberes pedagógicos, se van construyendo desde la experiencia; pero difícilmente se logra individualmente, ya que se requiere de la socialización con los compañeros en los diferentes espacios donde conviven, para con ello dar soporte a su quehacer.

Considerando que el profesional de la educación realiza una traducción del currículum formal al currículum real para hacer llegar 
los contenidos académicos a sus estudiantes, se rescata que, el dominio pleno del conocimiento científico - relacionado con las asignaturas que imparte - en la práctica no es determinante para el éxito de la clase, empero, se hacen indispensables las técnicas y dinámicas didácticas para trabajar dichos contenidos dentro del aula; cabe señalar que, no con ello quiere decir que es aceptable carecer del conocimiento científico del contenido que se está enseñando al impartir una clase. Por otro lado, el hecho de dominar la teoría de la asignatura no produce en automático la adquisición del conocimiento por parte del alumno. En este mismo sentido, Shulman (2005) expone que el docente retrospectivamente realiza un análisis de su quehacer, con la intención de reconstruir los sucesos, en otras palabras, esta reconstrucción se traduce en el aprendizaje que el docente adquiere a través de su experiencia.

\section{Discusión y conclusiones}

La práctica docente se interrelaciona con diversos elementos, que de acuerdo con Reyes et al. (2018), la hacen complicada, y esto obedece a todo aquello que envuelve el entorno educativo; sin embargo, este análisis crítico requiere de ir acompañado del ejercicio ordenado y metódico que tiene que ver con el análisis y la comprensión de las acciones, así como de un diálogo colectivo, que ayuda a los profesores a entender las dificultades y retos que se enfrenta en la cotidianeidad, también sensibilizarse acerca de los problemas educativos; pues si no es así, sería un obstáculo para modificar su labor cotidiana.

Lo anterior, coloca al profesor en condiciones óptimas para la transformación y profesionalización de la tarea que desarrolla, a fin de dar respuesta a las dificultades e impredecibilidades de las situaciones que ocurren en la realidad educativa. En este sentido, Guerrero (2016) considera que "la transformación permanente y sistemática de su práctica pedagógica es su finalidad, sin embargo, antes de lograrla hay que ir dando pauta a esa restructuración” (p. 17), inicialmente, reconociendo sus aciertos y errores.

En cada uno de los espacios donde el profesional en cuestión da vida a su quehacer se presenta un conjunto específico de relaciones, las cuales caracterizan el ejercicio de cada maestro, lo que significa que cada práctica es única, particular e irrepetible, esto obedece a la diversidad de su historia personal y profesional, aunado a las peculiaridades de las instituciones y de sus miembros. Por lo tanto, su actuar corresponde en parte a sus características personales, a su formación profesional, a las condiciones institucionales donde labora, y a las particularidades de sus estudiantes y demás agentes educativos. En congruencia con Tardif (2005), el ejercicio del profesional de la educación se encuentra estrechamente relacionada con cada una de las interacciones que establece con sus estudiantes durante la acción educativa.

El profesor se enfrenta diariamente a su labor como un ser impregnado de valores, quien a lo largo de su ejercicio ha construido su actuación, uniendo fragmentos de su vida personal y de su ejercicio académico, pero no solo eso, sino que va incluyendo además las vivencias de otros actores de la educación; por lo que se puede reconocer lo complejo que resulta examinar el ejercicio del docente debido a su carácter multifactorial; asimismo, Blanco (2013) manifiesta que las relaciones sociales desarrolladas en este ámbito posibilitan la oportunidad de comprender y reflexionar la práctica en cuestión, reconociendo la estrecha conexión existente entre esta y el discurso.

Con respecto a la construcción de saberes pedagógicos, no se puede obviar que el maestro requiere haber adquirido, a lo largo de su preparación profesional, una serie de conocimientos obtenidos a través de cursos ofertados por los diversos centros escolares; pero, se reconoce que la praxis es lo que lo corona como profesional de la educación; (Martínez et al., 2017); empero, Tardif (2014), afirma que "el cuerpo docente está devaluado en relación con los saberes que posee y transmite" (p. 26). La idea anterior, permite 
visualizar al profesional de la educación como el sujeto responsable de comprender e interpretar todos los elementos relacionados con la dinámica áulica, a fin de favorecer el aprendizaje en los estudiantes (Noguera et al., en Salazar, 2001).

La acción relacionada con la docencia no se limita al proceso de transmitir algún conocimiento, su actuación aborda situaciones relacionadas con los procesos cognoscitivos, afectivos, volitivos y conductuales de los estudiantes; de ahí que se requiere reconocer que la actividad en cuestión es desgastante tanto física como emocionalmente; tal realidad se refleja en la aportación de El Sahili (2011), donde afirma, que la complejidad de esta, está relacionada con el peso de los compromisos adquiridos desde su quehacer hasta los de tipo social dentro del mismo. Aunado a ello, Shulman (2005) considera que se requieren considerar los objetivos y las asignaturas abordadas en cada grado escolar, el entorno donde se desarrolla la clase, así como las particularidades de los estudiantes que forman parte de cada aula.

Independientemente de la historia o formación de los maestros, sus prácticas presentan una diversidad de matices que reflejan las capacidades, habilidades y saberes requeridos para atender las necesidades de los cambios sociales y educativos en cada centro escolar. Las condiciones laborales de cada uno son las que imprimen su sello en su quehacer, sin embargo, representan un gran desafío ya que cada grupo tiene personalidad propia; obviamente relacionada con el nivel educativo, el contexto sociocultural de sus estudiantes, etc.; personalidad que el profesor debe descifrar y entender para poner en marcha sus saberes con el fin de diseñar los espacios y condiciones necesarias para acercar los conocimientos a los alumnos. Empero, desde la visión de Herrán y González (en Rivera \& Hernández, 2017), "los docentes enseñan más por lo que son que lo saben" (p. 99).

A fin de concluir, se reconoce que el resultado de esta investigación aporta elementos teóricos que posibilitan un acercamiento al área de la práctica docente, insumos que permiten al profesorado la oportunidad de reconocer los significados que otorga a su ejercicio pedagógico, a fin de reconstruir las nociones y acciones de su labor, obedeciendo esto a la nueva lectura de su realidad. Se sugiere para futuras líneas de investigaciones abordar un análisis comparativo entre las percepciones y significados que ofrecen los profesores de los diferentes niveles educativos.

\section{Referencias bibliográficas}

Aguilar-Gavira, S., \& Barroso-Osuna, J.M. (2015). La triangulación de datos como estrategia en investigación educativa. [Data Triangulation As Education Researching Strategy]. PíxelBit. Revista de Medios y Educación, 47, 77-88. http://dx.doi.org/10.12795/pixelbit.2015.147.05

Blanco-López, G. (2013). Cambio de significado de la práctica docente a través de un eje diacrónico. RIDE Revista Iberoamericana para la Investigación y el Desarrollo Educativo, 10, 1-26. https://bit.ly/2FMjljK

Castillo-Bustos, M.R., \& Montoya-Rivera, J. (2015). Dinámica ideo-espiritual de la formación estético-pedagógica del docente. Alteridad, 10(2), 190-204. http://dx.doi.org/10.17163/alt.v10n2.2015.05

Davini, M.C. (2015). La formación en la práctica docente. Paidós.

Denzin, N.K., \& Lincoln, Y.S. (2011). El campo de la investigación cualitativa. Manual de Investigación Cualitativa Volumen II. Gedisa.

Denzin, N.K., \& Lincoln, Y.S. (2012). Paradigmas $y$ perspectivas en disputa. Manual de Investigación Cualitativa Volumen II. Gedisa.

Denzin, N.K., \& Lincoln, Y.S. (2013). Estrategias de investigación cualitativa. Manual de Investigación Cualitativa Volumen III. Gedisa.

Díaz-Quero, V. (2006). Formación docente, práctica pedagógica y saber pedagógico. Revista de Educación Laurus, 12, 88-103. https://bit. ly/35Tr9KN

Domingo-Roget, Á. (2013). Práctica reflexiva para docentes. De la reflexión ocasional a la reflexión metodológica. Publicia. 
El-Sahili, L.F. (2011). Docencia: Riesgos y desafíos. Trillas.

Evangelista-Díaz, R. (2015). La práctica en las políticas curriculares iberoamericanas para la formación docente. RMIE Revista Mexicana de Investigación Educativa, 20(65), 443-459. https://bit.ly/2Rgyhvs

Fierro, C., Fortoul, B., \& Rosas, L. (1999). Transformando la práctica docente. Paidós Mexicana.

González-Maura, V.L., López-Rodríguez, A., \& Valdivia-Díaz, J.E. (2017). Diplomado en docencia e investigación universitaria Una experiencia formativa para el desarrollo de competencias profesionales docentes en la Universidad de Atacama. Revista Iberoamericana de Educación 74, 121-146. https://doi.org/10.35362/rie740611

Guerrero-Calderón, M.A. (2016). Portafolios de experiencias docentes, el profesor como investigador de su práctica. RIDE Revista Iberoamericana para la Investigación y el Desarrollo Educativo, 7(13), 621-643. https://doi.org/10.23913/ride.v7i13.248

Hurtado-Espinoza, A.K., Serna-Antelo, A.M., \& Madueño-Serrano, M.L. (2015). Práctica docente del profesor universitario: Su contexto de aprendizaje. Profesorado. Revista de currículum y formación del profesorado, 19(2), 215-224. https://bit.ly/2Tm4mFc

Leiner, M. (febrero, 2005) Grupos focales: Una ventaja para el análisis cuantitativo. Avances Coordinación de investigación, 80. https://bit.ly/2BFnbMN

López-Aguilar, M.J. (2013). Una reforma «educativa» contra los maestros y el derecho a la educación. El Cotidiano, (179), 55-76. https://bit.ly/2QO7BTS

Loredo-Enríquez, J., Romero-Lara, R., \& Inda-Icaza, P. (2008). Comprensión de la práctica y la evaluación en el posgrado a partir de la percepción de los profesores. Revista Electrónica de Investigación Educativa, 1-16. https://bit.ly/2uMfkJJ

Martínez-Izaguirre, M., Álvarez de Eulate, C.Y., \& Villardón-Gallego, L. (2017). Competencias profesionales del profesorado de educación obligatoria. Revista Iberoamericana de Educación, 14, 171-192. https://doi.org/10.35362/rie740613

Montanares, E.G., \& Junod, P.A. (2018). Creencias y prácticas de enseñanza de profesores universitarios en Chile. Revista Electrónica de Investigación Educativa, 20(1), 93-103. https://doi.org/10.24320/redie.2018.20.1.1383

Perrenoud, P. (2001). Desarrollar la práctica reflexiva en el oficio de enseñar. Profesionalización y razón pedagógica. GRAÕ. https://bit.ly/2MDc2xT

Quecedo, R., \& Castaño, C. (2002). Introducción a la metodología de investigación cualitativa. Revista de Psicodidáctica, (14), 5-39. https://bit.ly/35LLSjX

Reyes-Piñuelas, E.P., González-Machado, E.C., \& Be-Ramírez, P.A. (2018). Evaluar la práctica docente a partir de los comentarios de estudiantes universitarios. Revista Iberoamericana de Educación, 76(2), 117-134. https://doi.org/10.35362/rie7623096

Rivera-Morales, A., \& Hernández-Durán, G. (2017). $\mathrm{El}$ autoconcepto de docentes universitarios. Revista Iberoamericana de Educación, (73)2, 87-100.

https://doi.org/10.35362/rie732213

Rodríguez-Ebrard. L.A. (2016). La investigaciónacción como instrumento de evaluación de la propia práctica docente. IE Revista de Investigación Educativa de la REDIECH, 7(12), 52-59. https://bit.ly/2uMfGA3

Rodríguez-Gómez, G., Gil-Flores, \& García-Jiménez, E. (1996). Metodología de la investigación cualitativa. Aljíbe. https://bit.ly/3eR88h8

Salazar, J.M. (2001). El aprendizaje en los docentes y su integración a la práctica de aula. Perspectiva Educacional, Formación de Profesores, 54(1), 55-74. https://bit.ly/35L1h3L

Sandoval-Casilimas, C.A. (2002). Investigación cualitativa. ARFO Ltda. https://bit.ly/2UdZbqu

Shulman, L.S. (2005). Conocimiento y enseñanza: Fundamentos de la Nueva Reforma Educativa. Profesorado. Revista de currículum $y$ formación del profesorado, 9(2). https://bit.ly/3cwh8GR

Tardif, M. (2005). Los saberes del docente y su desarrollo profesional. Narcea, S. A. de Ediciones.

Terrón-Bañuelos, A. (2019). Enseñar, y pensar la profesión: Comentarios a la autobiografía de un docente (José María Rozada Martínez). 
[Teaching and reflecting on the profession: Comments on a teacher's autobiography (José María Rozada Martínez)]. Con-Ciencia Social, 2, 163-172. https://bit.ly/2tUhOp2

Torres-Rivera, A.D., Badillo-Gaona, M., ValentínKajatl,N.O., \& Ramírez-Martínez,E.T. (2014). Las competencias docentes: El desafío de la educación superior. Innovación Educativa, 14(66), 129-145. https://bit.ly/2TiU1tl

Tovar-Gálvez, J.C., \& García-Contreras, G.A. (2012). Investigación en la práctica docente universitaria: Obstáculos epistemológicos y alternativas desde la Didáctica General Constructivista. Educ. Pesqui. 38(4), 881-895. http://dx.doi.org/10.1590/S1517-97022012000400007

Verástegui-Martínez, M. (2019). El conocimiento educativo de los docentes en la transformación y la mejora educativa. En Monarca, H., Gorostiaga, J.M., \& Pericacho-Gómez, F.J. Calidad de la educación: Aportes de la investigación y la práctica (pp. 171-192). Dykinson. https://bit.ly/2B0xnPe

Vergara-Fregoso, M. (2016). La práctica docente. Un estudio desde los significados. [Teaching practice. A study from the meanings]. Revista Cumbres 2(1), 73-99. https://bit.ly/2QPgMDL

Vergara Fregoso, M. (2005). Significados de la práctica docente que tienen los profesores de educación primaria. REICE-Revista Electrónica Iberoamericana sobre Calidad, Eficacia $y$ Cambio en Educación, 3(1), 685-697. https://bit.ly/35SL38S

Villalpando-Sifuentes, C.G. (2015). La transposición pragmática como práctica de significación en educación primaria. Borderland Studies Publishing House.

Vitarelli, M.F. (Comp.). (2005). Formación docente $e$ investigación (Propuestas en desarrollo). Ediciones LAE Laboratorio de Alternativas Educativas.

Zabalza-Beraza, M.Á. (2012). El estudio de las buenas prácticas docentes en la enseñanza universitaria. Revista de Docencia Universitaria, 10(1), 17-42.

https://doi.org/10.4995/redu.2012.6120 